\title{
Multiple Integrations of Human T-Lymphotropic Virus Type 1 Proviruses in the Engrafted Cells from the Asymptomatic Carriers in NOD/SCID/ $\gamma c^{\text {null }}$ Mice
}

\author{
Ikuo Yamamoto $^{\mathrm{a}}$ Ichiro Takajo $^{\mathrm{a}}$ Kazumi Umeki $^{\mathrm{a}}$ Kazuhiro Morishita ${ }^{\mathrm{b}}$ \\ Kinta Hatakeyamac Hiroaki Kataokad Hajime Nomura ${ }^{a}$ Akihiko Okayama $^{\text {a }}$ \\ ${ }^{a}$ Department of Rheumatology, Infectious Diseases and Laboratory Medicine, ${ }^{b}$ Division of Tumor and Cellular

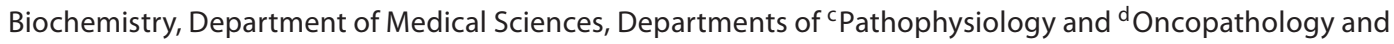 \\ Regenerative Biology, University of Miyazaki, Miyazaki, Japan
}

\section{Key Words}

Human T-lymphotropic virus type $1 \cdot$ NOG mouse $\cdot$

Multiple proviral integration

\begin{abstract}
Objectives: Successful engraftment of human T-lymphotropic virus type 1 (HTLV-1)-infected cells and a marked increase of proviral DNA loads (PVLs) in non-obese diabetic/ severe combined immunodeficient (NOD/SCID) $/ \gamma c^{\text {null }}$ (NOG) mice have been reported. Whether the increased PVL in transplanted mice is due to the new infection of HTLV-1 was examined. Methods: Mononuclear cells from 3 NOG mice with primary engraftment from asymptomatic HTLV-1 carriers were transplanted into a second group of NOG mice. HTLV-1 PVL, proviral integration by fluorescence in situ hybridization assay, expression of viral antigen, and T-cell clonality were analyzed. Results: The PVLs in the secondarily transplanted NOG mice were significantly higher than those of primarily transplanted NOG mice. Multiple signals of HTLV-1 proviruses in the nucleus of the infected cells were revealed by fluorescence in situ hybridization analysis. Expression of HTLV-1 tax/rex mRNA and antigen was observed. The variety of T-cell clones was limited in the transplanted NOG mice. Conclusions: Multiple proviral integrations were
\end{abstract}

considered to be due to the new infection from HTLV-1-infected cells to the other cells. Only a certain fraction of T cells seemed to have selectively survived in NOG mice after engraftment.

Copyright $\odot 2010$ S. Karger AG, Basel

\section{Introduction}

Human T-cell leukemia virus type 1 (HTLV-1) is the causative agent of adult T-cell leukemia/lymphoma (ATL), and a progressive demyelinating disease known as HTLV-1-associated myelopathy/tropical spastic paraparesis [1-4]. As HTLV-1 infection is associated with cellto-cell contact, the major routes of transmission of HTLV1 are via breast-feeding, sexual contact, and blood transfusion [5-7]. It has been postulated that the clonal proliferation of HTLV-1 infected T cells likely plays an important role in the maintenance of HTLV-1 infection in carriers over long periods of time $[8,9]$.

A variety of animal models of HTLV-1 infection have provided available information about HTLV-1 infection in vivo [10]. Recently, genetically modified mice, including both transgenic and knockout mice, have become important models for testing the behavior of human cells in 
vivo. Non-obese diabetic (NOD)/severe combined immunodeficient (SCID) mice, such as the NOD/LtSz-scid and the NOD/Shi-scid mouse, have been considered appropriate models for this purpose [11-14]. We have shown the successful engraftment of HTLV-1-infected cells from asymptomatic carriers in NOD/SCID/ $/ \gamma c^{\text {null }}$ (NOG) mice [15]. In this previous study, HTLV-1 provirus was recognized only in the human mononuclear cells, which infiltrated into the various organs of transplanted NOG mice. The increased proviral DNA loads $\geq 100,000$ copies per 100,000 human cells were shown. This proviral DNA load increasing may be due to clonal expansion of HTLV1 -infected cells directly derived from the peripheral blood of asymptomatic carriers. Alternatively, transmission of HTLV-1 from the infected human cells to other human cells might occur in vivo. To clarify whether these speculations are true or not, we isolated the human cells in NOG mice with primary engraftment from HTLV-1 asymptomatic carriers, and inoculated them into the other NOG mice. The integration of HTLV-1 provirus into the chromosome of human cells was examined by fluorescence in situ hybridization (FISH) assay. To evaluate whether there is an active replication of HTLV-1 or not, the expression of tax/rex mRNA and HTLV-1 antigen was also tested. In addition, the rearrangement of the T-cell receptor- $\gamma(\mathrm{TCR}-\gamma)$ gene that reflects the clonality of $\mathrm{T}$ cells [16] was compared between mice with primary and secondary engraftment. The results suggested that the transmission of HTLV-1 from infected cells to other cells occurred efficiently in NOG mice and only limited fraction of $\mathrm{T}$ cells seemed to have selectively survived.

\section{Materials and Methods}

\section{Subjects}

Three asymptomatic HTLV-1 carriers (carrier A, B, and C) were recruited into this study after obtaining written informed consent. The white blood cell counts of the HTLV-1-infected carriers were within the reference value without any abnormal cells. The peripheral blood mononuclear cells (PBMCs) were isolated from carriers $\mathrm{A}, \mathrm{B}$, and $\mathrm{C}$ by density gradient centrifugation on Histopaque-1077 (Sigma-Aldrich, St. Louis, Mo., USA), washed three times with phosphate-buffered saline (PBS) and preserved with Cell banker ${ }^{\circledR}$ (JUJI Inc., Tokyo, Japan) at $-80^{\circ}$ until use. The study protocol was approved by the Institutional Review Board of University of Miyazaki.

Inoculation of Human PBMCs from HTLV-1 Carriers into the NOG Mice

NOG mice were purchased from the Central Institute of Experimental Animals (Kawasaki, Japan). All mice were bred and maintained under specific pathogen-free conditions in the De- partment of Bioresources, Division of Biotechnology, Frontier Science Research Center, University of Miyazaki (Miyazaki, Japan). Female NOG mice aged from 8 to 10 weeks were used for the experiments. A total of $5 \times 10^{6}$ human PBMCs from carriers A, $B$, and $C$ were injected intraperitoneally into one half of them and intravenously into the other half (primary transplantation, NOG A-1, B-1, and C-1, respectively). The mice were sacrificed 4 weeks after inoculation. Mononuclear cells were isolated from the livers of NOG A-1, B-1, and C-1 using density gradient centrifugation. More than $99 \%$ of these mononuclear cells were T cells (data not shown). A total of $3 \times 10^{6}$ cells were inoculated into new NOG mice (secondary transplantation, NOG A-2, B-2, and C-2, respectively). The secondarily transplanted NOG mice were sacrificed 4 weeks after inoculation. The experimental protocol was approved by the University of Miyazaki ethics review committee for animal experimentation.

Quantification of Human Cells and HTLV-1 Provirus

Chromosomal DNA was isolated from the PBMCs of carriers and mononuclear cells obtained from the livers of NOG mice by sodium dodecyl sulfate proteinase $\mathrm{K}$ digestion at $56^{\circ}$, followed by phenol-chloroform extraction and ethanol precipitation. HTLV-1 proviral copy number (i.e. proviral DNA load) was measured by real-time polymerase chain reaction (PCR) using LightCycler ${ }^{\circledR}$ DX 400 (Roche Diagnostics, Mannheim, Germany) as described previously [15]. In brief, quantitative real-time PCR was performed for human albumin DNA, mouse GAPDH DNA, and HTLV-1 provirus. The primers and probe for the human albumin gene were as follows: the forward primer Alb-S (5'-GCTGTCATCTCTTGTGGGCTGT-3'), the reverse primer Alb-AS (5'-AAACTCATGGGAGCTGCTGGT- ${ }^{\prime}$ ), and the FAM-labeled albumin TaqMan ${ }^{\circledR}$ probe (5'-FAM-CCTGTCATGCCCACACAAATCTCTCC-TAMRA-3'). TaqMan Gene Expression Assays (Applied Biosystems Japan, Tokyo, Japan) were used for the primers and the probe for the mouse GAPDH gene. The primers and the probe for the $p X$ region of HTLV-1 were as follows: the forward primer pX2-S (5'-CGGATACCCAGTCTACGTGTT-3': positions 2359-2379), the reverse primer pX2-AS (5'-CAGTAGGGCGTGACGATGTA-3': positions 7458-7439), and the FAM-labeled pX2 probe (FAM-CTGTGTACAAGGCGACTGGTGCC-TAM$\mathrm{RA}^{\prime}$ : positions 7386-7408). Quantitative PCR was performed in a duplicate manner.

FISH Analysis of HTLV-1-Infected Cells

FISH analysis was performed to detect HTLV-1 proviral signals in the mononuclear cells isolated from the livers of NOG mice according to a slightly modified version of the method reported by Taniwaki et al. [17]. For FISH assay probes, PCR products prepared using four different primer sets for HTLV-1, which cover almost the entire genome of HTLV-1, were used. Primer sets used for PCR were as follows: region 1: the forward primer (HTLV23F 5'-TGACAATGACCATGAGCCCCAA-3': positions 23-44), and the reverse primer (HTLV2060R 5'-CTAATAGGAGGGCATCTTCCTC-3': positions 2060-2039), region 2: the forward primer (HTLV2013F 5'-AGCCCACTATCCCAGAACCAGA-3': positions 2013-2034), and the reverse primer (HTLV4741R 5'-CGAATAGCAAGGGAGGTACACA-3': positions 4741-4720), region 3: the forward primer (HTLV4562F 5'AGACACCTTTTCAGGAGCCATC-3': positions 4562-4583), and the reverse primer (HTLV7292R 5'-AGGGCTGTTTCGAT- 
GCTTGCCT-3': positions 7292-7271), and region 4: the forward primer (HTLV7150F 5'-CTTCTAAGGATAGCAAACCGTCA$3^{\prime}$ : positions 7150-7172), and the reverse primer (HTLV9041R 5'CTCTCCTGAGAGTGCTATAGGA-3': positions 9041-9020). Subsequently, the PCR products were subcloned by pGEM $^{\circledR}{ }_{-} \mathrm{T}$ Easy vector system (Promega, Madison, Wisc., USA) and were supplied for the probe preparation. The Nick Translation System $^{\circledR}$ (Invitrogen, Carlsbad, Calif., USA) and Biotin-16-dUTP ${ }^{\circledR}$ (Roche) were used for biotinylating probes. Cell suspensions on glass slides were fixed in methanol/acetic acid (3:1). Biotinylated probe DNA was denatured, allowed to preanneal with unlabeled human Cot-l DNA (15-30 pg) at $37^{\circ}$ for $20 \mathrm{~min}$, and hybridized with treated chromosomal samples on glass slide at $37^{\circ}$ for $20 \mathrm{~h}$. Biotinylated probes were detected with Fluorescein Avidin DCS ${ }^{\circledR}$ (Vector Laboratories, Inc., Burlingame, Calif., USA), Biotinylated Anti-Avidin $\mathrm{D}^{\circledR}$ (Vector Laboratories, Inc.), and a second layer of Fluorescein Avidin DCS. Cells were counterstained with 4',6-diamino-2-phenylindole (DAPI) and mounted in VECTASHIELD $^{\circledR}$ (Vector Laboratories, Inc.). Results were analyzed on a conventional fluorescence microscope DMRA (Leica, Wetzlar, Germany) and photographed by a Leica Q550CW system (Leica, Cambridge, UK). Hybridization signals were evaluated in approximately 100 nuclei.

Detection of HTLV-1 mRNA by Reverse Transcriptase-PCR

The detailed procedure for reverse transcriptase (RT)-PCR was described elsewhere [15]. In brief, total cellular RNA was extracted from the liver of transplanted NOG C mice using TRIzol ${ }^{\circledR}$ Reagent (Invitrogen) according to the manufacturer's instructions. Five $\mu \mathrm{g}$ of total RNA was reverse transcribed using M-MCV RT (Invitrogen) using random primers. The following primers were used for RT-PCR:PX-1 (5'-AGGGTTTGGACAGAGTCTTC3': positions 7335-7354) and PX-2 (5'-AAGGACCTTGAGGGTCTTAG-3': positions 7590-7571) for tax/rex mRNA; GAPDH118F (5'-GTCGGAGTCAACGGATTTGGTCG-3': positions 7698)and GAPDH-640R(5'-CATGGACTGTGGTCATGAGTCC-3': positions 598-577) for GAPDH mRNA (Sigma-Aldrich Japan). The PCR products were electrophoresed on $1.2 \%$ agarose gel, and visualized by ethidium bromide staining. The RNA sample from an HTLV-1-positive cell line, HUT102, was used as a positive control at a 1:100 dilution.

Detection of HTLV-1 Antigen by Indirect Immunofluorescence Assay

Mononuclear cells isolated from the liver of transplanted NOG $\mathrm{C}$ mice were deposited on the glass slides using Cytospine 3 (Shandon Scientific, London, UK). Cell suspensions on glass slides were fixed in cold acetone for $1 \mathrm{~min}$, air dried, and treated with $10 \%$ bovine serum albumin in PBS for $30 \mathrm{~min}$. Then, they were reacted with 1:100 diluted human serum with or without anti-HTLV-1 antibody at $37^{\circ}$ for $30 \mathrm{~min}$, washed with PBS, and treated with fluorescein isothiocyanate-conjugated goat anti-human IgG/ $\mathrm{F}\left(\mathrm{ab}^{\prime}\right)_{2}$ (Medical \& Biological Laboratories, Nagano, Japan) at $37^{\circ}$ for $30 \mathrm{~min}$. Cells were counterstained with DAPI and were analyzed on a conventional fluorescence microscope DMRA and photographed by a Leica Q550CW system.

Analysis of Rearranged TCR- $\gamma$ Gene

The rearrangement of TCR- $\gamma$ gene of mononuclear cells in transplanted NOG mice was detected by PCR based on the report by Benhattar et al. [16], using forward primer (TVG 5'-AGGGTTGTGTTGGAATCAGG-3') and reverse primer (TJG 5'-CGTCGACAACAAGTGTTGTTCCAC- $3^{\prime}$ ). The expected size of the PCR products was approximately $170-200$ base pairs. Amplified products were electrophoresed on $10 \%$ nondenaturing polyacrylamide gel, and visualized by ethidium bromide staining. Then, PCR products were subcloned by PGEM $^{\circledR}$-T Easy vector system (Promega) and transfected into Escherichia coli JM109 (TAKARA Biosystems, Shiga, Japan). The reaction mixture was applied to a Luria-Bertani (LB) agar plate containing ampicillin and incubated overnight at $37^{\circ}$. From each subject, 30-40 colonies were randomly obtained and cultured by LB broth containing ampicillin overnight at $37^{\circ}$. DNA sequences of the cloned PCR products were analyzed using the ABI PRISM Big Dye Terminator Cycle Sequencing Ready Reaction kit (Applied Biosystems, Foster City, Calif., USA) and an ABI PRISM 310 Genetic Analyzer (Applied Biosystems).

\section{Statistical Analysis}

The Kruskal-Wallis test was used to compare the proviral DNA loads among the PBMCs of the carriers, and the engrafted cells in NOG mice with primary and secondary transplantation. One-tailed t test was used to compare the percentage of human cells among total cells in the livers of NOG mice with primary and secondary transplantation. The $\chi^{2}$ test was used to compare frequencies of clones with unique rearrangement of the V-J region of TCR- $\gamma$ among tested PCR products in carriers and NOG mice with primary engraftment and secondary engraftment. $\mathrm{p}$ values $<0.05$ were considered as statistically significant.

\section{Results}

\section{HTLV-1 Proviral Loads in NOG Mice with Primary and Secondary Transplantation}

All mice grew normally without piloerection or weight loss for 1 month after transplantation. No tumors or lymph node swelling were found when the mice were sacrificed. Infiltration of mononuclear cells was seen in various organs of all tested NOG mice on microscopic examination, as described previously [15]. In order to measure the number of human cells, mouse cells, and HTLV-1 provirus present in the tissues of NOG mice, human albumin DNA, mouse GAPDH DNA, and HTLV-1 provirus were quantified using real-time PCR. HTLV-1 proviral DNA copy numbers in 100 human cells and the percentages of human cells among total cells in the livers of transplanted NOG mice are shown in figure 1. HTLV1 proviral copies in 100 PBMCs of carriers A, B, and C were $12.3,3.2$, and 0.2 , respectively. The proviral loads in the livers of primarily transplanted NOG mice (NOG A-1, B-1, and C-1) were higher (82.6, 134.7, and 11.1 copies per 100 human cells, respectively) than those of carriers $\mathrm{A}, \mathrm{B}$ and C. Moreover, the proviral loads in the livers of secondarily transplanted NOG mice (NOG A-2, B-2, and C-2) were significantly higher $(1,428.6,445.3$, and 812.0 
Fig. 1. HTLV-1 proviral DNA loads and percentage of human cells in the livers of transplanted NOG mice. a HTLV-1 proviral copies in 100 mononuclear cells. b Percentage of human cells among total cells in the liver of NOG mice. Open circles, closed circles and squares indicate NOG A series, NOG B series and NOG C series, respectively.

Fig. 2. Representative data of FISH assay of HTLV-1 proviruses integrated into the genome of the mononuclear cells in the liver of NOG mice with secondary engraftment. Green signals of HTLV-1 proviruses by fluorescein isothiocyanate can be detected in the nucleus of a cell dyed in blue by diamidino phenylindole. a No signal. b Two signals. c Four signals. d Thirteen signals. $\times 1,000$.
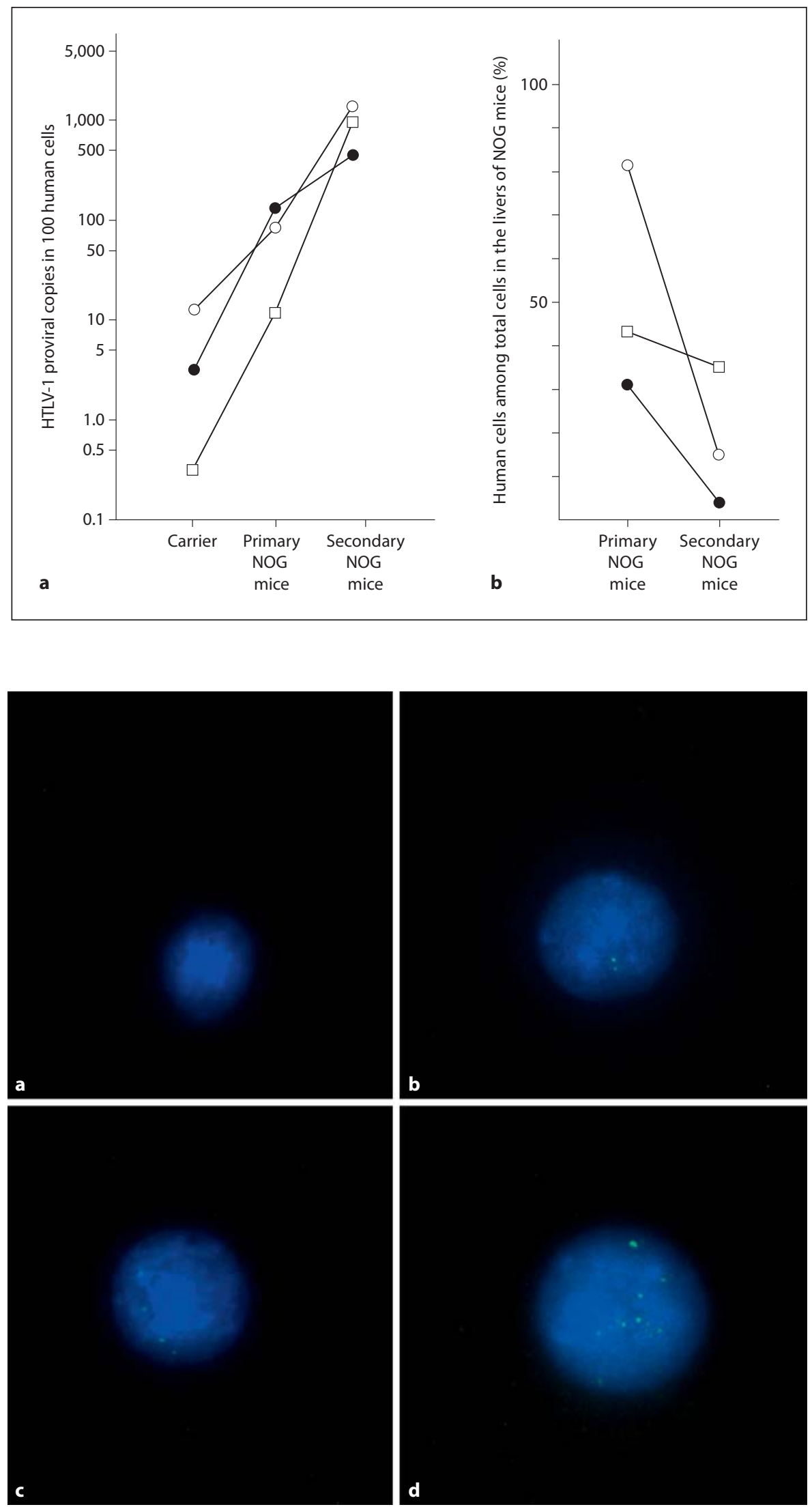

Yamamoto/Takajo/Umeki/Morishita/ Hatakeyama/Kataoka/Nomura/Okayama 


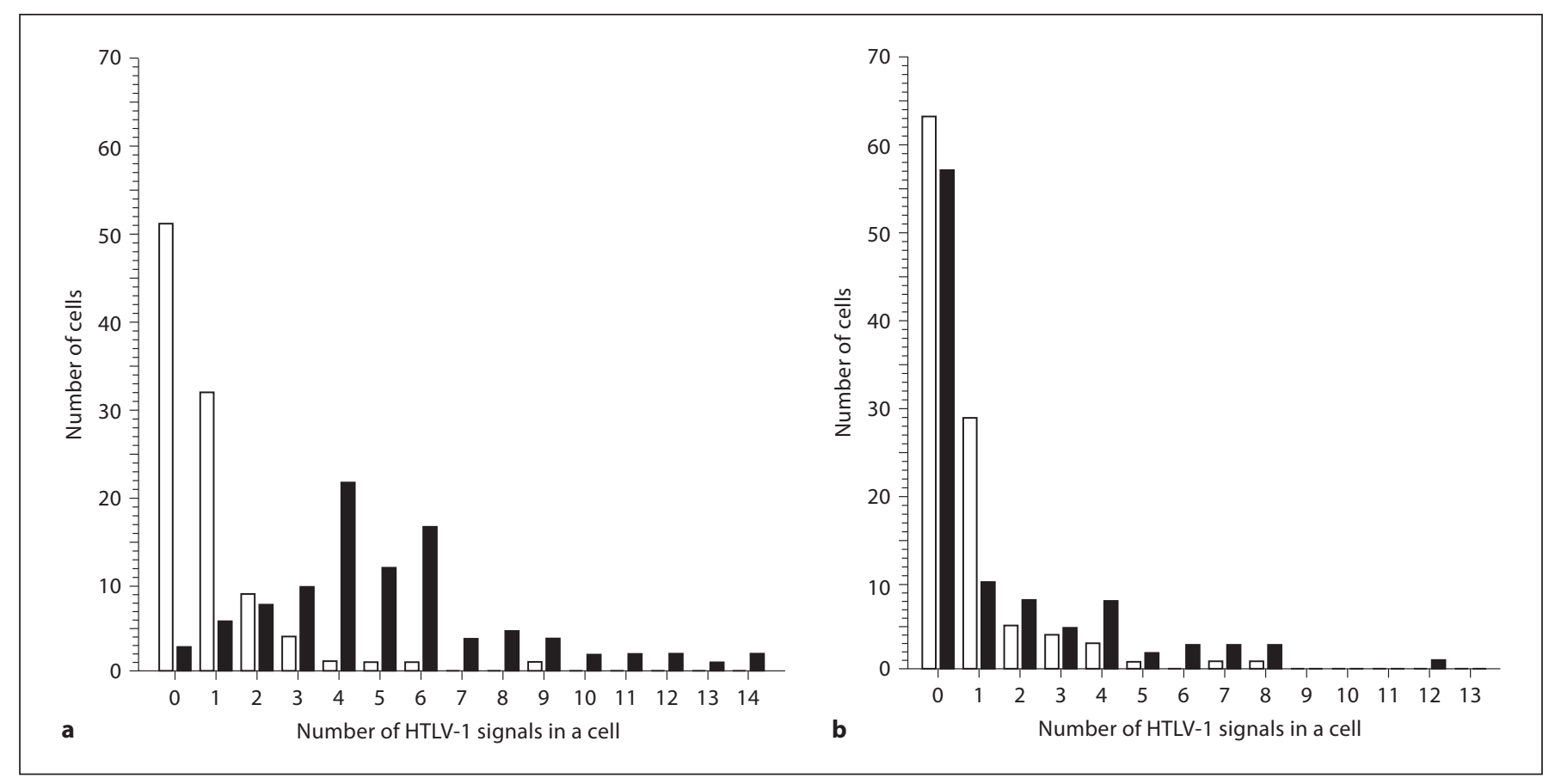

Fig. 3. Distributions of the numbers of cells based on the number of signals of HTLV-1 proviruses by FISH assay in the transplanted NOG mice. a NOG A series. b NOG B series. Open and closed columns represent the primarily and secondarily transplanted NOG mice, respectively.

copies per 100 human cells, respectively) than those of primarily transplanted NOG mice (NOG A-1, B-1, and $\mathrm{C}-1 ; \mathrm{p}=0.039$, Kruskal-Wallis test). The percentage of human cells among total cells in the livers of secondarily transplanted NOG mice (NOG A-2: 16.5\%, B-2: 4.6\%, and C-2: $35.6 \%)$ were significantly lower than those of primarily transplanted NOG mice (NOG A-1: 81.9\%, B-1: $30.2 \%$, and C-1: $43.3 \% ; \mathrm{p}=0.035$, one-tailed $\mathrm{t}$ test). These results suggested that HTLV-1-infected cells at least in the secondarily transplanted NOG mice harbor multiple copies of HTLV-1 proviruses; however, the number of the human cells in the secondarily transplanted NOG mice decreased compared to the primarily transplanted NOG mice. Therefore, it was assumed that more than several copies of HTLV-1 proviruses were integrated into the genome of infected cells.

\section{FISH Analysis of HTLV-1-Infected Cells Infiltrated in} the Livers of Primarily and Secondarily Transplanted NOG Mice

Because the proviral loads especially increased in secondarily transplanted NOG mice suggested multiple integrations of proviruses in HTLV-1-infected cells, HTLV1-infected cells in the livers of NOG A and B mice with primary and secondary engraftment were analyzed by FISH assay. NOG C mice could not be analyzed due to insufficient samples for FISH analysis. When the PBMCs of HTLV-1 carriers were analyzed by FISH, cells with only one signal, which represented one HTLV-1 provirus, were observed occasionally among cells with no signal. No cells with multiple signals were observed (data not shown). When cells isolated from the liver of primarily and secondarily transplanted NOG mice were analyzed, the numbers of signals per cell varied from 0 to 14 (fig. 2). The distributions of numbers of cells based on the number of signals by FISH in the primarily and secondarily transplanted NOG mice are shown in figure 3. The fraction of cells with no signal were most prevalent (approximately $50 \%$ of all cells) in the primarily transplanted mouse, NOG A-1. Approximately $30 \%$ and fewer than $20 \%$ of all cells were found to have one signal and multiple signals in one cell, respectively. In the secondarily transplanted mouse, NOG A-2, the fraction of cells with no signal was only $3 \%$ and greater than $90 \%$ of cells had multiple signals of HTLV-1 proviruses. The primarily and secondarily transplanted mice, NOG B-1 and B-2, respectively, showed the same trend, although the trend was less evident compared to that of NOG A-1 and A-2. 


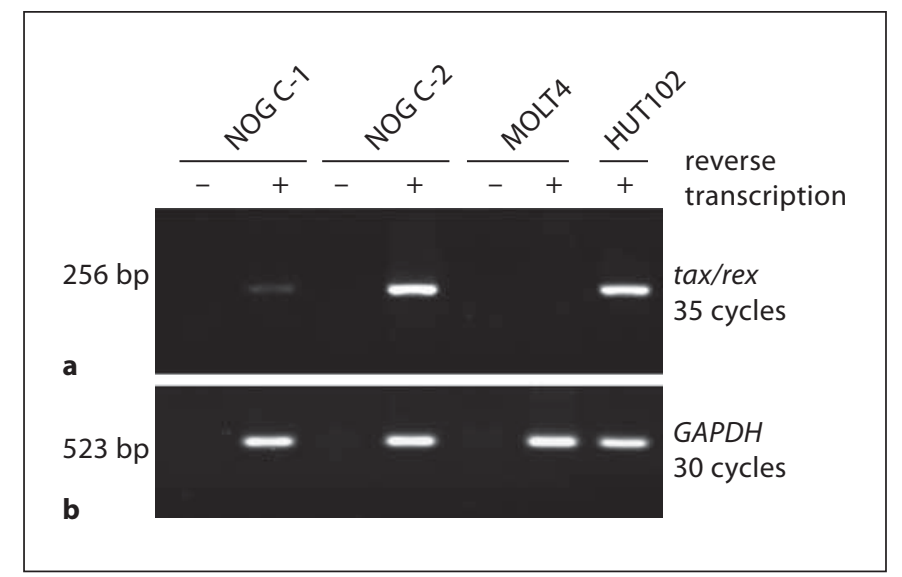

Fig. 4. Detection of the HTLV-1 tax/rex mRNA in the livers of transplanted NOG mice (C-1 and C-2) by RT-PCR. Upper panel: HTLV-1 tax/rex mRNA; lower panel: GAPDH mRNA (normalizing RNA samples). MOLT 4 and HUT102 cells were used as the HTLV-negative and -positive control, respectively.

\section{Detection of HTLV-1 mRNA in Liver of NOG Mice by} RT-PCR

To evaluate whether there is an active replication of HTLV-1 in the transplanted NOG mice, HTLV-1 tax/rex mRNA was tested by RT-PCR. As shown in figure 4, HTLV-1 tax/rex mRNA was shown in the livers of transplanted NOG C-1 and C-2. The signal from the secondary transplanted mouse, $\mathrm{C}-2$, looked stronger than that from the firstly transplanted mouse, $\mathrm{C}-1$.

\section{Detection of HTLV-1 Antigen by IF Assay}

Because the transcription of HTLV-1 gene in the transplanted NOG mice was shown in the previous experiment (fig. 4), HTLV-1 antigen (viral protein) on the mononuclear cells from the livers in the transplanted NOG mice was tested. As shown in figure 5, HTLV-1 antigen was positive for mononuclear cells both in NOG C-1 and C-2 mice by IF assay. Thirteen cells (6.5\%) and 104 cells (52\%) among 200 cells counted tested positive for HTLV-1 antigen in NOG C-1 and C-2 mice, respectively. These results met the results of HTLV-1 tax/rex RNA (fig. 4), which showed stronger signal in C-2 mouse than that in $\mathrm{C}-1$ mouse.

\section{Analysis of the Clonality of Human T Cells Using}

Detection of Rearrangement of TCR- $\gamma$ Gene in

Primarily and Secondarily Transplanted NOG Mice

Whether the human $\mathrm{T}$ cells, which survived in the transplanted NOG mice, are polyclonal or oligoclonal is the next question. The clonality of HTLV-1-infected cells in the carriers has been commonly analyzed by methods that identify cells with identical HTLV-1 provirus integration sites using Southern blot or the techniques based on PCR. Because multiple proviruses were shown to be integrated into the genome of HTLV-1-infected cells in NOG mice with engraftment, we could not analyze T-cell clonality using the method based on the HTLV-1 provirus integration sites in this case. Therefore, we used the method to analyze the rearrangement of the $\mathrm{V}$-J region of TCR- $\gamma$ gene of T cells. As shown in figure 6, the products of PCR to amplify the V-J region of TCR- $\gamma$ gene both from the HTLV-1-positive and -negative PBMCs showed a smear pattern in electrophoresis assay, which reflected the polyclonality of $\mathrm{T}$ cells. On the other hand, secondarily transplanted NOG mice (NOG A-2, B-2, and C-2) showed several dense bands, which indicated the clear oligoclonal pattern. The primarily transplanted NOG mice, especially NOG B-1 and C-1, showed a moderate pattern between HTLV-1 carriers and the secondarily transplanted NOG mice. Therefore, only restricted clones of human T cells seemed to have survived in the transplanted NOG mice.

The frequencies of $\mathrm{T}$ cell clones with a unique (different) V-J region of TCR- $\gamma$ gene in each NOG mouse with primary and secondary engraftments were assessed by cloning and DNA sequencing of the PCR products (fig. 7). Only two clones with unique rearrangement of the V-J region of TCR- $\gamma$ gene were detectable more than twice among 31 tested PCR products in the PBMCs of carrier A (fig. 7a). In the primarily transplanted NOG mouse (NOG A-1), five clones with unique rearrangement of the V-J region of TCR- $\gamma$ gene were detectable more than twice among 35 tested PCR products, and the frequencies of these clones were greater than those of carrier A. In the secondarily transplanted NOG mouse (NOG A-2), four clones with unique rearrangement of the V-J region of TCR- $\gamma$ gene were detectable more than twice among 33 tested PCR products, and the frequencies of these clones were greater than those of NOG A-1. This tendency was also evident in cases of the NOG mice with primary and secondary engraftments from carrier B and C (fig. 7b, c, respectively). The proportions of clones with unique rearrangement of the V-J region of TCR- $\gamma$ gene among tested cloned PCR products were 28/31 (90\%), 25/35 (71\%), and $16 / 33$ (49\%) in carrier A, NOG A-1, and NOG A-2, respectively. The rates of unique clone in the case of carrier $\mathrm{B}$, NOG B-1, and NOG B-2 were 27/28 (96\%), 21/33 (64\%), and 14/38 (37\%), respectively, and those in carrier C, NOG C-1, and NOG C-2 were 31/32 (97\%), 27/34 (79\%), 
NOG C-1

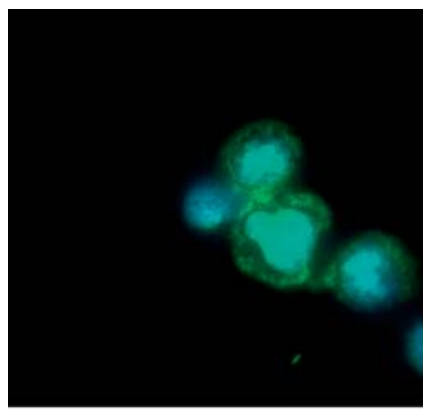

a

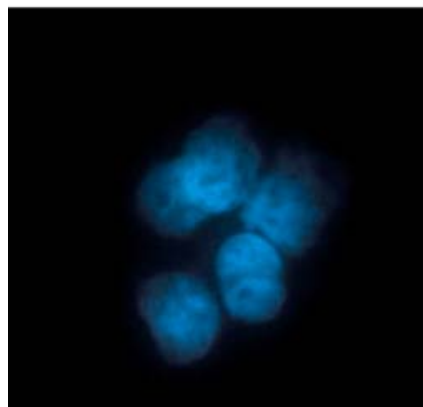

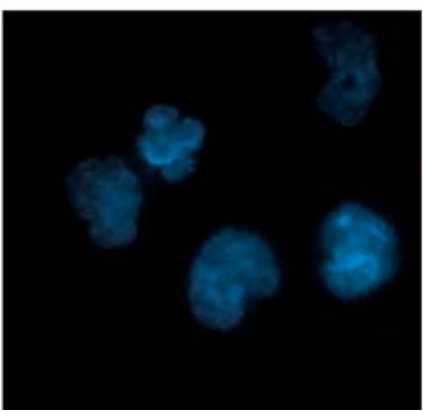

MOLT4
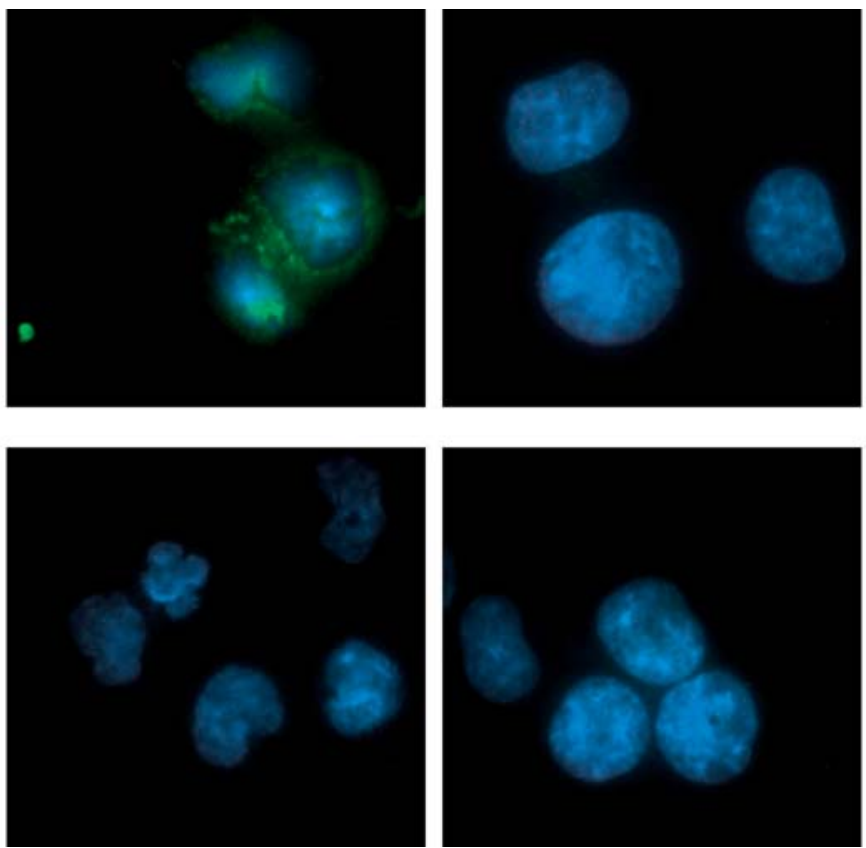

HUT102
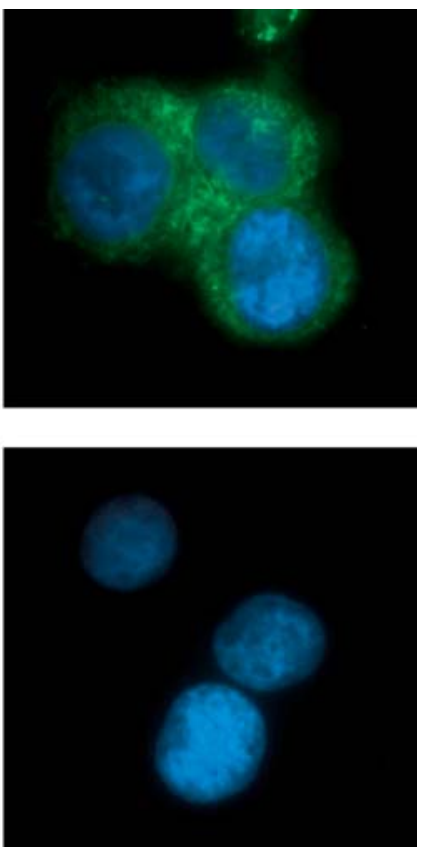

Fig. 5. Detection of HTLV-1 antigen in the mononuclear cells from the livers of transplanted NOG mice by indirect immunofluorescence assay. Mononuclear cells from the livers of transplanted NOG C-1 and NOG C-2 mice were reacted with the sera from HTLV-1-positive and -negative subjects. MOLT4 and
HUT102 cells were used as the HTLV-negative and -positive control, respectively. Magnification: NOG C- 1 and NOG C- $2 \times 1,000$, MOLT4 and HUT102 ×640. a Anti-HTLV-1 antibody-positive serum used as 1st antibody. b Anti-HTLV-1 antibody-negative serum as control.

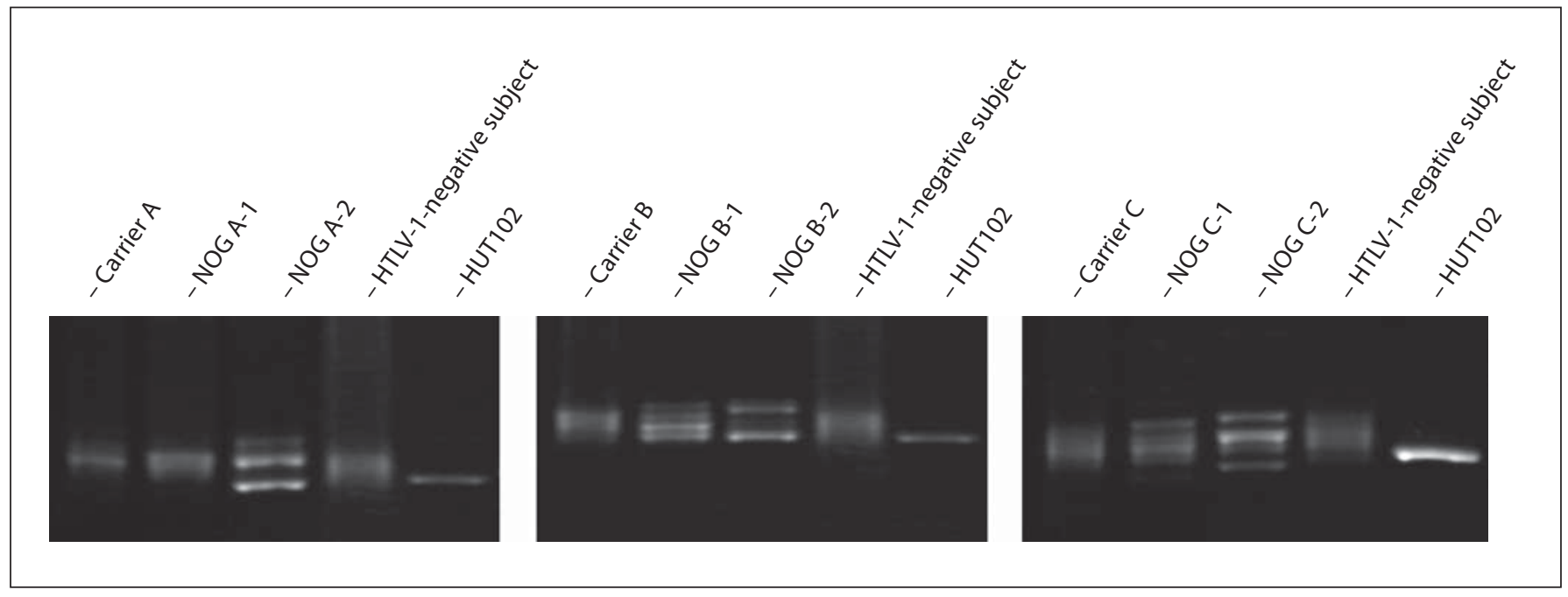

Fig. 6. PCR analysis of the rearrangement of V-J region of TCR- $\gamma$ gene. Panels represent 10\% polyacrylamide gel electrophoresis of PCR products from the PBMCs of carriers and the human cells in the livers of primarily and secondarily transplanted NOG mice.
PBMCs from an HTLV-1-negative subject and HTLV-1-positive cell line, HUT102 were used as negative and positive controls, respectively. 


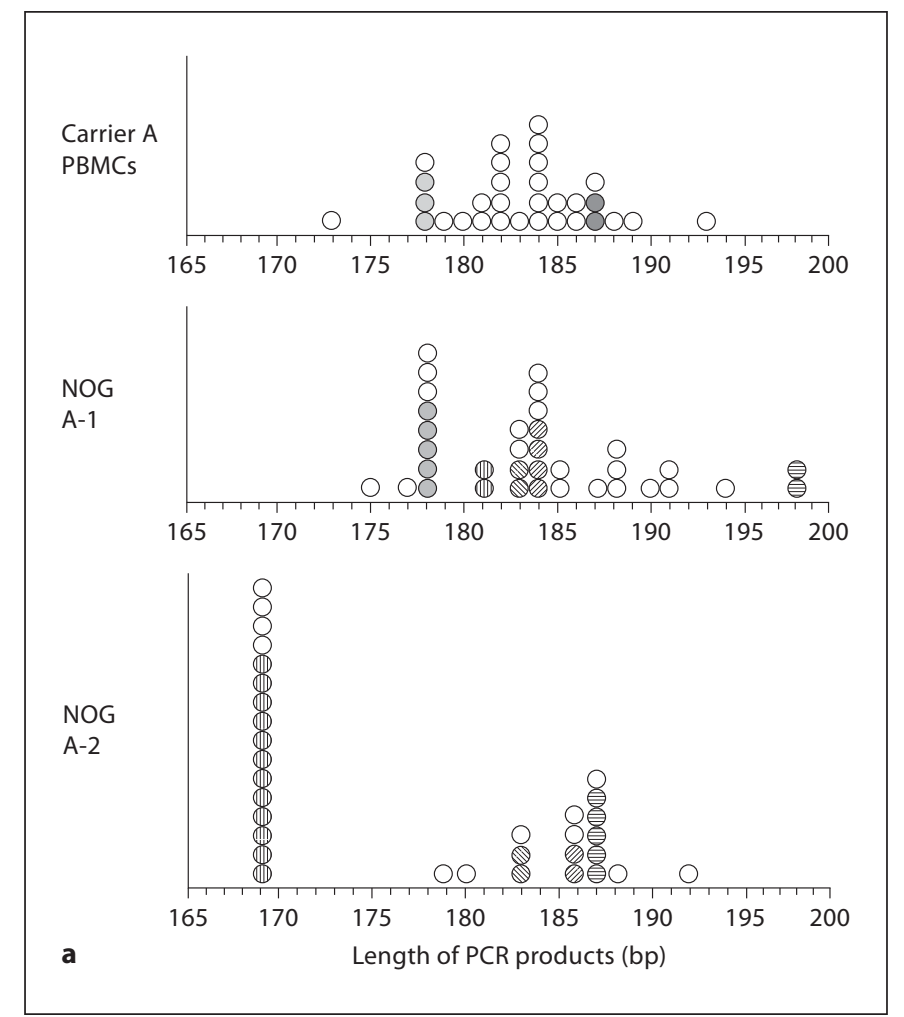

Fig. 7. Frequencies of T cells with unique (different) V-J region of TCR- $\gamma$ gene in the carriers' PBMC and the NOG mice with the primary and secondary engraftments. a-c Results from NOG mice A, B and C, respectively. The circles with the same pattern indicate cloned PCR products with identical V-J region of TCR- $\gamma$ gene and open circles indicate cloned PCR products with unique V-J region of TCR- $\gamma$ gene. Arrows indicate clones with identical V-J region of TCR- $\gamma$ gene, which were detected both in the NOG mice with the primary and the secondary engraftment.
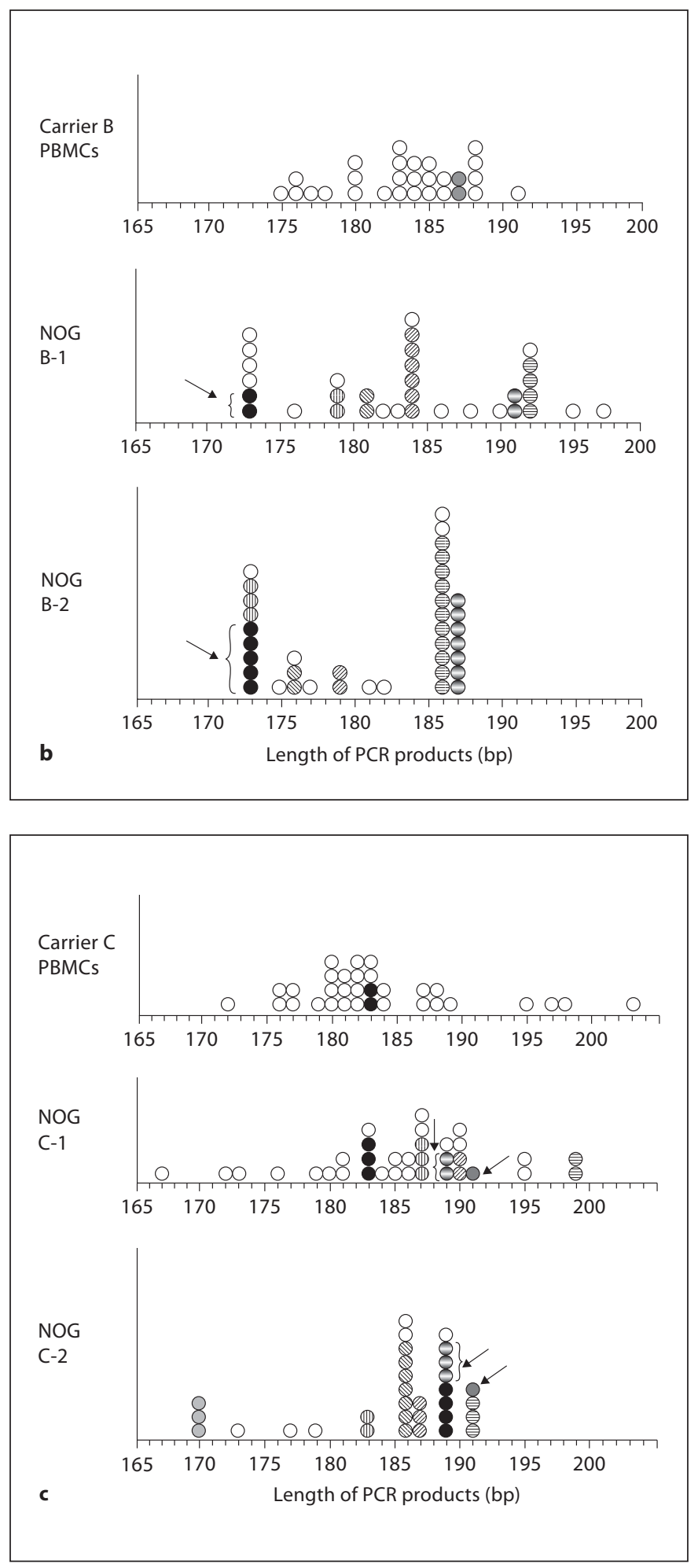
Table 1. Frequencies of clones with unique rearrangement of $\mathrm{V}-\mathrm{J}$ region of T-cell receptor- $\gamma$ gene among tested PCR products in carriers, NOG mice with primary engraftment and secondary engraftment

\begin{tabular}{llll}
\hline & $\begin{array}{l}\text { Carriers' } \\
\text { PBMCs }\end{array}$ & $\begin{array}{l}\text { NOG mice } \\
\text { with primary } \\
\text { engraftment }\end{array}$ & $\begin{array}{l}\text { NOG mice } \\
\text { with secondary } \\
\text { engraftment }\end{array}$ \\
\hline NOG A series & $28 / 31(90 \%)$ & $25 / 35(71 \%)$ & $16 / 33(49 \%)$ \\
NOG B series & $27 / 28(96 \%)$ & $21 / 33(64 \%)$ & $14 / 38(37 \%)$ \\
NOG C series & $31 / 32(97 \%)$ & $27 / 34(79 \%)$ & $14 / 32(44 \%)$ \\
\hline Total & $86 / 91(95 \%)$ & $73 / 102(72 \%)$ & $44 / 103(43 \%)$ \\
& \multicolumn{2}{c}{ a } & \multicolumn{2}{c}{ b } \\
& \multicolumn{2}{c}{ c }
\end{tabular}

${ }^{a} \mathrm{p}<0.0001$ for carriers' PBMCs vs. NOG mice with primary engraftment; ${ }^{\mathrm{b}} \mathrm{p}<0.0001$ for NOG mice with primary engraftment vs. NOG mice with secondary engraftment; ${ }^{c} p<0.0001$ for carriers' PBMCs vs. NOG mice with secondary engraftment.

and 14/32 (44\%), respectively. The differences in the frequencies of clones with unique rearrangement of the $\mathrm{V}-\mathrm{J}$ region of TCR- $\gamma$ gene among tested cloned PCR products from carriers and NOG mice with primary and secondary engraftments were statistically significant $(\mathrm{p}<0.0001$, $\chi^{2}$ test; table 1). Therefore, the clonality of $\mathrm{T}$ cells in the NOG mice was less heterogeneous than that of HTLV-1 carriers, especially in the secondarily transplanted NOG mice. In addition, none of the clones were consistently detected among carrier A, NOG A-1, and NOG A-2. Only one clone in the NOG B series and two clones in the NOG $\mathrm{C}$ series were detected both in the primarily and secondarily transplanted mice (arrows in fig. $7 \mathrm{~b}, \mathrm{c}$ ).

\section{Discussion}

In the present study, successful engraftment of HTLV1-infected cells, which were isolated from the infiltrated organs of NOG mice with primary transplantation of PBMCs from asymptomatic HTLV-1 carriers, was shown in the NOG mice with secondary transplantation. HTLV1 proviral copies per cell significantly increased in the secondarily transplanted NOG mice when compared with those in the PBMCs of original carriers and, even with those in the primarily transplanted NOG mice. The HTLV-1 proviruses were confirmed to be integrated into the human chromosome only and not into the mouse chromosome in our previous study [15]. The integration of more than one copy of HTLV-1 provirus per human cell in the transplanted mice was expected based on the numbers of HTLV-1 proviruses obtained by real-time PCR. Indeed, FISH analysis showed that more than one signal, which represented the integrated HTLV-1 proviruses in nuclei, were detectable in the cells of the transplanted NOG mice in the present study. A greater number of cells with multiple signals were found in the NOG mice with secondary engraftment than in the NOG mice with primary engraftment. Because only one signal per cell was detectable in the PBMCs of the HTLV-1 carriers, the cells with multiple proviruses might be caused by the new infection of HTLV-1 from infected cells to the other cells in NOG mice. Indeed, HTLV-1 tax/rex mRNA and HTLV-1 antigen were detected in these cells, indicating an active replication of HTLV-1. The signal of HTLV-1 tax/rex mRNA in NOG C-2 mouse was stronger than that in C-1 mouse. Moreover, the number of HTLV-1 antigenpositive cells in NOG C-2 mouse was more than that in NOG C-1 mouse. These results suggested HTLV-1 replication in the secondarily transplanted mouse was more active than that in the firstly transplanted mouse.

Alternatively, there is a possibility that there are small numbers of HTLV-1-infected cells with multiple integrations of proviruses, undetectable by FISH assay, in PBMCs of HTLV-1 carriers, and these cells expanded in the NOG mice after the transplantation. The integration of two or three copies of proviruses in the leukemic cells of some patients with ATL has been reported [18]. However, integration of more than 10 copies of proviruses into the genome of human cells in vivo has not been reported to date. Moreover, with the exception of one or two clones, same T-cell clones were not found both in the mice with primary and secondary engraftments. Therefore, the cells with multiple proviruses found in the transplanted NOG mice were less likely due to the expansion of the HTLV-1 cells, which were directly derived from the original carriers.

The reason why the new infection from HTLV-1-infected cells to the other cells occurred after transplantation in the NOG mice was not clear. There is no antibody to HTLV-1 or effective cytotoxic T cells in NOG mice. The inhibition of HTLV-1 infection to the offspring by the sera of HTLV-1 carriers was reported in the rabbit model [19]. The addition of antibody to HTLV-1, anti-gp46, to the coculture of HTLV-1-infected and -uninfected cells also inhibits new infection [20]. These data suggest that immune responses to HTLV-1 may prevent active infection between noninfected cells and infected cells in 
carriers. Therefore, loss of normal immune response in the NOG mice may account for the new infection of HTLV-1. Alternatively, immune cells in the PBMCs from HTLV-1 carriers, which were inoculated into the NOG mice, recognized the tissue of NOG mice as a foreign antigen and were activated. HTLV-1-infected T cells among these cells were also activated during this process and might have been triggered for the production of the viral antigen. The results in the present study suggest that the new cell-to-cell infection of HTLV-1 is able to occur even in humans when the HTLV-1 carriers are under strong immunosuppression. Indeed, HTLV-1 infection from the recipient cells to the donor cells was reported in the case of bone marrow transplantation to patients with ATL [21].

The human cells which survived in the NOG mice were decreased in number, especially in the mice with secondary transplantation. When the rearrangement of TCR- $\gamma$ gene of human cells was analyzed in the present study, the variety of T-cell clones was limited in the NOG mice. These data suggest that only a certain fraction of $\mathrm{T}$ cells selectively survived in NOG mice after the transplantation. The majority of the mononuclear cells isolated from the NOG A-2 were shown to harbor HTLV-1 provirus by FISH. HTLV-1 Tax protein has been shown to promote the proliferation of infected cells [22-25]. Therefore, it was possible that the HTLV-1-infected T cells had a growth advantage due to Tax protein in the NOG mice because the transcription of HTLV-1 $p X$ genes was shown in the present study. However, half of the cells from the NOG B-2 were shown not to harbor HTLV-1 provirus by FISH assay. The direct activation of T cells as an immune response to the mouse antigens is also possible. Alternatively, activated HTLV-1-infected cells due to Tax protein may produce cy tokines to induce the activation of HTLV1-noninfected cells (bystander effect) in NOG mice.
Whether the selectively surviving T cells in NOG mice have characteristics similar to leukemic cells is an interesting question. When smoldering-type ATL cells were transplanted into NOD/SCID/beta 2 -microglobulin ${ }^{\text {null }}$ mice, expansion of single HTLV-I-infected clone was reported [22]. However, based on the analysis of the rearrangement of TCR- $\gamma$ gene in the present study, the majority of the T-cell clones found in the primarily transplanted NOG mice disappeared in the secondarily transplanted NOG mice. Further study is necessary to clarify whether the surviving T cells in NOG mice have the potential to develop leukemic cells and whether this engraftment model is useful in the identification of carriers with higher risk of developing ATL.

In conclusion, HTLV-1-infected cells derived from NOG mice with primary engraftment were successfully transplanted into other NOG mice. An increased number of HTLV-1 proviral copies per cell was observed in the secondarily transplanted NOG mice, and this was considered due to the new infection from HTLV-1-infected cells to the other cells. The human cells which survived in NOG mice were decreased in number, especially in mice with secondary engraftment. Only a fraction of $\mathrm{T}$ cells seemed to have selectively survived in NOG mice after the transplantation. This engraftment model using NOG mice could be useful in clarifying the mechanism of HTLV-1 infection in vivo and the development of the reagents to inhibit HTLV-1 infection.

\section{Acknowledgements}

We thank Ms. Y. Motoyoshi (University of Miyazaki) for her technical assistance. This study was supported by a grant-in-aid from the Ministry of Education, Science, Sports and Culture, Japan, and Miyazaki Prefecture Collaboration of Regional Entities for the Advancement of Technological Excellence, JST.

\section{References}

1 Uchiyama T, Yodoi J, Sagawa K, Takatsuki K, Uchino H: Adult T-cell leukemia: clinical and hematologic features of 16 cases. Blood 1977;50:481-492.

$\checkmark 2$ Yoshida M, Miyoshi I, Hinuma Y: Isolation and characterization of retrovirus from cell lines of human adult T-cell leukemia and its implication in the disease. Proc Natl Acad Sci USA 1982;79:2031-2035.

\3 Osame M, Usuku K, Izumo S, Ijichi N, Amitani $\mathrm{H}$, Igata $\mathrm{A}$, Matsumoto $\mathrm{M}$, Tara $\mathrm{M}$ : HTLV-1 associated myelopathy, a new clinical entity. Lancet 1986;327:1031-1032.

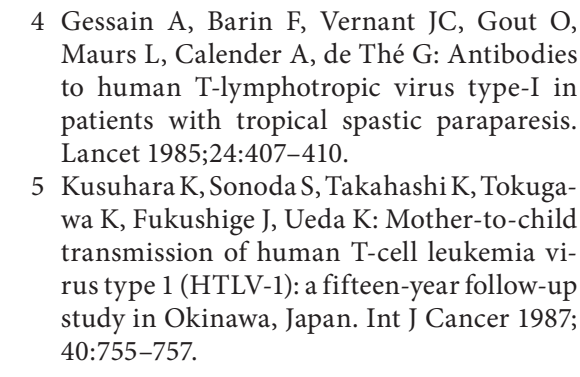
Gessain A, Barin F, Vernant JC, Gout O to human T-lymphotropic virus type-I in patients with tropical spastic paraparesis. Lancet 1985;24:407-410.

5 Kusuhara K, Sonoda S, Takahashi K, Tokugawa K, Fukushige J, Ueda K: Mother-to-child transmission of human T-cell leukemia vistudy in Okinawa, Japan. Int J Cancer 1987; 40:755-757.

\footnotetext{
6 Tajima K, Tominaga S, Suchi T, Kawagoe T, Komoda H, Hinuma Y, Oda T, Fujita K: Epidemiological analysis of the distribution of antibody to adult T-cell leukemia-virus-associated antigen: possible horizontal transmission of adult T-cell leukemia virus. Gann 1982;73:893-901.

7 Okochi K, Sato H, Hinuma Y: A retrospective study on transmission of adult T-cell leukemia virus by blood transfusion: seroconversion in recipients. Vox Sang 1984;46: 245-253.
} 
8 Etoh K, Tamiya S, Yamaguchi K, Okayama A, Tsubouchi H, Ideta T, Mueller N, Takatsuki K, Matsuoka M: Persistent clonal proliferation of human T-lymphotropic virus type 1-infected cells in vivo. Cancer Res 1997;57: 4862-4867.

-9 Cavrois M, Leclercq I, Gout O, Gessain A, Wain-Hobson S, Wattel E: Persistent oligoclonal expansion of human T-cell leukemia virus type 1-infected circulating cells in patients with tropical spastic paraparesis/ HTLV-1 associated myelopathy. Oncogene 1998;17:77-82.

10 Lairmore MD, Silverman L, Ratner L: Animal models for human T-lymphotropic virus type 1 (HTLV-1) infection and transformation. Oncogene 2005;24:6005-6015.

-11 Greiner DL, Shultz LD, Yates J, Appel MC, Perdrizet G, Hesselton RM, Schweitzer I, Beamer WG, Shultz KL, Pelsue SC, Leif JH, RajanTV: Improved engraftment of human spleen cells in NOD/LtSz-scid/scid mice as compared with C.B-17-scid/scid mice. Am J Pathol 1995;146:888-902.

-12 Shultz LD, Schweitzer PA, Christianson SW Gott B, Schweitzer IB, Tennent B, McKenna S, Mobraaten L, Rajan TV, Greiner DL, Leiter EH: Multiple defects in innate and adaptive immunologic function in NOD/LtSz-scid mice. J Immunol 1995;154:180-191.

13 Ueda T, Tsuji K, Yoshino H, Ebihara Y, Yagasaki H, Hisakawa H, Mitsui T, Manabe A, Tanaka R, Kobayashi K, Ito M, Yasukawa K, Nakahara T: Expansion of human NOD/ SCID-repopulating cells by stem cell factor, Flk2/Flt3 ligand, thrombopoietin, 1L-6, and soluble 1L-6 receptor. J Clin Invest 2000;105: 1013-1021.
14 Koyanagi Y, Tanaka Y, Tanaka R, Misawa N, Kawano Y, Tanaka T, Miyasaka M, Ito M, Ueyama Y, Yamamoto N: High levels of viremia in hu-PBL-NOD-scid mice with HIV-1 infection. Leukemia 1997;11(suppl 3):109112.

15 Takajo I, Umeki K, Morishita K, Yamamoto I, Kubuki Y, Hatakeyama K, Kataoka H, Okayama A: Engraftment of peripheral blood mononuclear cells from human Tlymphotropic virus type 1 carriers in NOD/ SCID $/ \gamma c^{\text {null }}$ (NOG) mice. Int J Cancer 2007; 121:2205-2211.

16 Benhattar J, Delacretaz F, Martin P, Chaubert $\mathrm{P}$, Costa J: Improved polymerase chain reaction detection of clonal T-cell lymphoid neoplasms. Diagn Mol Pathol 1995;4:108112.

17 Taniwaki M, Speicher MR, Lengauer C, Jauch A, Popp S, Cremer T: Characterization of two marker chromosomes in a patient with acute nonlymphocytic leukemia by two-color fluorescence in situ hybridization. Cancer Genet Cytogenet 1993;70:99-102.

18 Kamihira S, Dateki N, Sugahara K, Yamada Y, Tomonaga M, Maeda T, Tahara M: Realtime polymerase chain reaction for quantification of HTLV-1 proviral load: application for analyzing aberrant integration of the proviral DNA in adult T-cell leukemia. Int J Hematol 2000;72:79-84.
19 Miyoshi I, Takehara N, Sawada T, Iwahara Y, Kataoka R, Yang D, Hoshino H: Immunoglobulin prophylaxis against HTLV-I in a rabbit model. Leukemia 1992;6(suppl 1):2426.

20 Ishizaki J, Okayama A, Kuroki M, Tsubouchi $\mathrm{H}$ : Detection of human T-lymphotropic virus type I (HTLV-I) infection during coculture of HTLV-I infected and uninfected cells using inverse long PCR. Intervirology 2002; 45:164-171.

21 Ljungman P, Lawler M, Åsjo B, Bogdanovic G, Karlsson K, Malm C, McCann SR, Ringdén O, Gahrton G: Infection of donor lymphocytes with human $\mathrm{T}$ lymphotrophic virus type 1 (HTLV-I) following allogeneic bone marrow transplantation for HTLV-I positive adult T-cell leukaemia. Br J Haematol 1994;88:403-405.

22 Kawano N, Ishikawa F, Shimoda K, Yasukawa M, Nagafuji K, Miyamoto T, Baba E, Tanaka T, Yamasaki S, Gondo H, Otsuka T, Ohshima K, Shultz LD, Akashi K, Harada M; Efficient engraftment of primary adult T-cell leukemia cells in newborn NOD/SCID/ $/ 2$ microglobulin $^{\text {null }}$ mice. Leukemia 2005; 19 : 1384-1390.

23 Franchini G, Fukumoto R, Fullen JR: T-cell control by human T-cell leukemia/lymphoma virus type 1. Int J Hematol 2003;78:280296.

-24 Grassmann R, Aboud M, Jeang KT: Molecular mechanisms of cellular transformation by HTLV-1 Tax. Oncogene 2005;24:59765985.

-25 Yoshida M: Multiple viral strategies of HTLV-1 for dysregulation of cell growth control. Annu Rev Immunol 2001;19:475496. 